(C) 2016 IEEE. Personal use of this material is permitted. Permission from IEEE must be obtained for all other uses, in any current or future media, including reprinting/republishing this material for advertising or promotional purposes, creating new collective works, for resale or redistribution to servers or lists, or reuse of any copyrighted component of this work in other works. 


\title{
On the deviation of metamaterial spheres from effective medium
}

\author{
M. Lapine ${ }^{1,2}$, C. G. Poulton ${ }^{1}$ and R. C. McPhedran ${ }^{3}$ \\ ${ }^{1}$ School of Mathematical and Physical Sciences, University of Technology Sydney, 2007 NSW, Australia \\ ${ }^{2}$ Department of Nanophotonics and Metamaterials, ITMO University, Saint-Petersburg 197101, Russia \\ ${ }^{3}$ School of Physics, University of Sydney, 2006 NSW, Australia \\ mlapine@physics.usyd.edu.au
}

\begin{abstract}
We analyse the convergence of the actual properties of finite subwavelength metamaterial samples where all the prerequisites for a reliable effective medium description, except for the finite size, are fulfilled, towards the effective medium predictions. We show that the convergence is rather slow and it is likely that hundreds of thousands of individual meta-atoms must be assembled together before the properties of the resulting structure can be claimed to correspond to those a bulk material. These observations are directly relevant for practical design of metamaterials and their future development.
\end{abstract}

\section{INTRODUCTION}

All the most important claims in metamaterial research, that being either negative refraction with associated perfect lensing, or cloaking and optical transformation [1], or non-reciprocity [2], or nonlinerarity [3], were made exploiting a reference to their effective material parameters. It is then known that a number of precautions [4] must be observed before such parameters are reliably introduced [5, 6], and most of those precautions are, although still not widely followed, are well established [7]. And yet there is still an aspect which has been deprived of sufficient attention so far albeit having a core practical relevance: the peculiar features of finite samples of metamaterials, that is, all the practically relevant designs. We have previously demonstrated an important distinction between the theoretically predicted parameters and the actual properties of finite samples $[8,9]$. In this contribution, we offer a further physical insight into that distinction, studying the properties of highly symmetric metamaterial structures where all other effects but finites size are excluded, and analyse the slow convergence of their properties towards the effective medium predictions as the size of the samples grows.

\section{COMPARISON OF THE FINITE METAMATERIAL SPHERES TO EFFECTIVE MEDIUM PREDICTIONS}

For a finite system under a given excitation, the induced currents and hence the magnetisation can be calculated directly [10] taking all the mutual interactions between the loops into account. To exclude the shape effects as much as possible, we use the most simple shape of metamaterial samples, which is a sphere. We consider a series of metamaterial samples having a cubic lattice, truncated to a shape as close to a sphere as possible for a given size. The two different possibilities, arising from the structure of a cubic sample, are the flat and ragged options [9]. Clearly, for small spheres with just a few unit cells along the diameter the shape is remarkably ragged, however larger spheres (starting from around 10 lattice constants in diameter) appear reasonably smooth overall.

Imposing a uniform time-varying magnetic field along one of the axes of the structure, we evaluate the magnetisation along the same axis, the results presented in Fig. 1. Small discrete samples show remarkable deviations and less trivial frequency dependence with some extra resonances, however convergence towards the continuous model improves with size and becomes a clear trend for sizes above 10, and the results for the spheres of 15 and larger appear quite similar to each other; initially ragged configuration shows somewhat better convergence compared to a flat one. Even so, there is still a difference between large discrete spheres and the continuous one, which may be attributed to the effect of the elements closest to the boundary, which do not form a perfect spherical surface but a corrugated one, with the spherical approximation becoming increasingly good with size. While we have no computational tools to calculate much larger samples, we hypothesise that eventually the difference between a discrete sphere and a continuous one can be eliminated to good precision. 

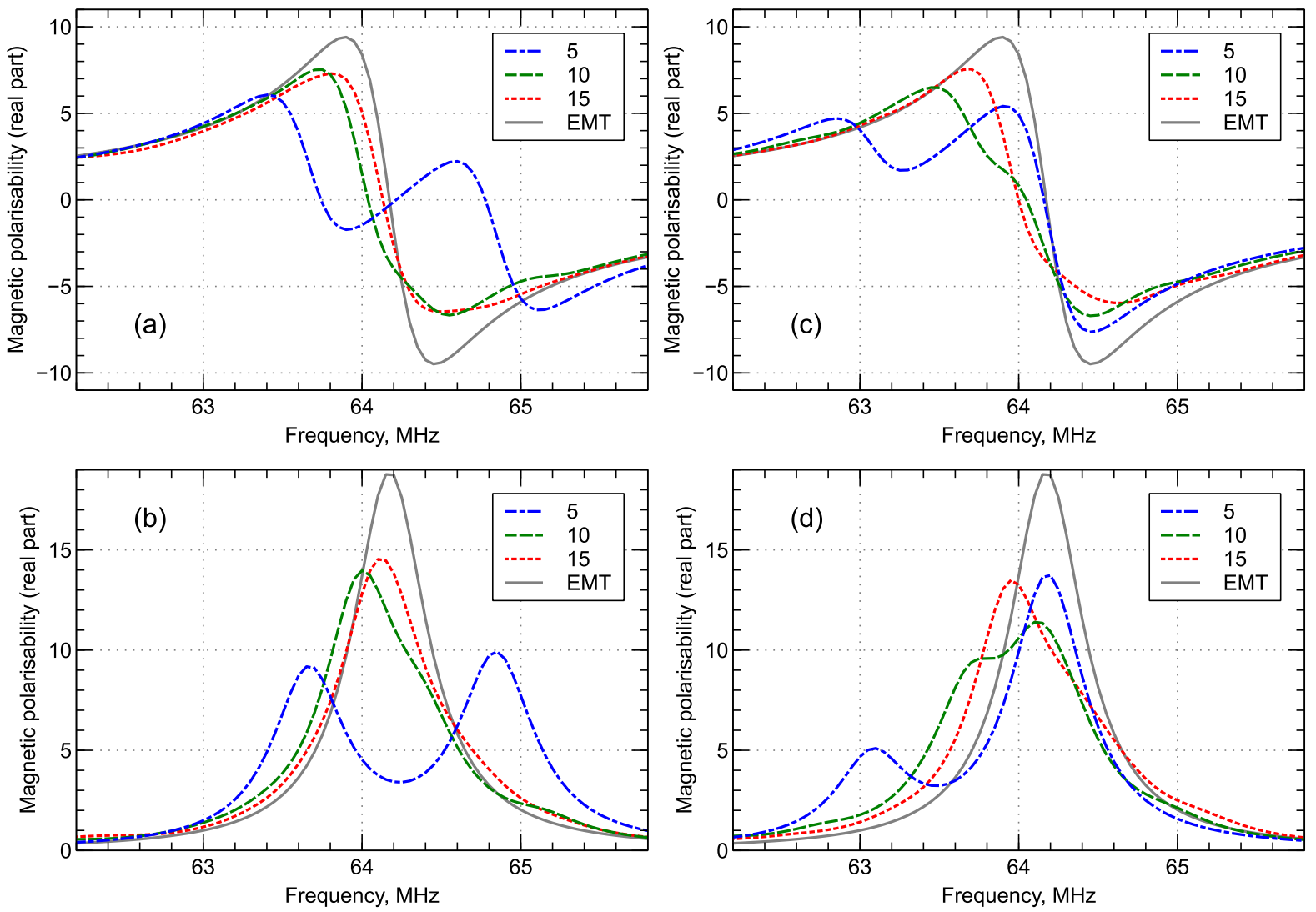

Fig. 1: Frequency dependence of real $(a, c)$ and imaginary $(b, d)$ parts of the magnetic polarisability (total magnetic moment per unit volume per unit field applied) of the spherical isotropic metamaterial samples obtained by truncating cubes with either ragged $(a, b)$ or flat $(c, d)$ configuration of the initial boundary. The panels include the results for spheres of different size $(5,10$ or 15 unit cells per diameter), as indicated in the insets. The grey curve ("EMT") shows the polarisation theoretically calculated for a homogeneous sphere with the effective permeability [11] corresponding to the considered metamaterial structure.

For a quantitative insight into the convergence towards the the effective medium predictions, we analyse the difference between the frequency of the resonance of the magnetic polarisability, observed for finite spheres, and the resonance predicted with the effective medium theory [11] for a bulk material with the corresponding metaatoms and lattice parameters (Fig. 2). Note that the theoretical calculation for the uniaxial structure [11] is still valid for each of the tensorial components in the analogous isotropic one. For anisotropic samples with uniaxially oriented rings, the convergence is particularly clear, revealing an inverse proportionality between the size and the frequency offset. Importantly, the same trend is observed for a low-loss anisotropic structure, where the data are much more scattered but are best fitted with a very similar dependence. With the isotropic samples, the initially ragged spheres show a great similarity to the anisotropic case, whereas the initially flat ones indeed demonstrated a somewhat slower convergence. With the low-loss isotropic cases, the inverse proportionality is less convincing, however we assume that in these cases the size is still too small to make a reliable analysis.

\section{CONCLUSIONS}

The effects outlined above are particularly prominent in metamaterials with strongly interacting elements [12], such as those based on ring resonators. It also appears that having a resonance is essential for boundary effects to spread through the structure. Indeed, as opposed to resonant metamaterials, response of artificial diamagnetics of finite size and discrete structure is in a good agreement with the effective medium predictions. Our conclusions [13] are likely to be applicable in a wide frequency range and for many specific designs, so these results may have severe implications for practical development of metamaterials. 


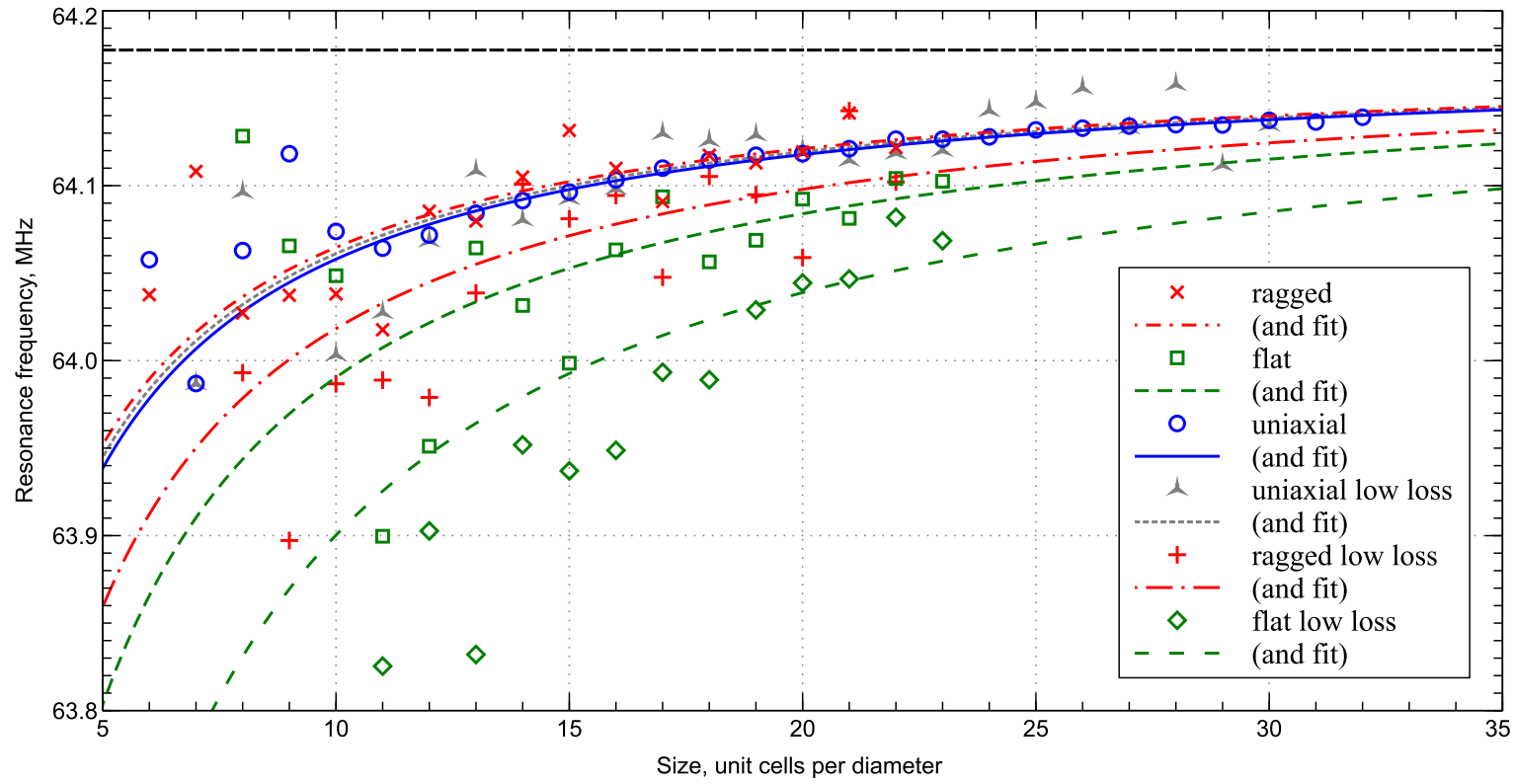

Fig. 2: Convergence of the magnetic polarisability of finite discrete metamaterial spheres, towards that of a homogeneous sphere as predicted by effective medium theory (shown as the dashed horizontal line at ca. $64.177 \mathrm{MHz}$ ). The results are presented for anisotropic uniaxial as well as for the isotropic structures truncated from either ragged or flat boundary type of the initial cubes. All the cases are taken with either regular or low (10\% of the regular) dissipation. The best fits for the frequency shift assuming an inverse proportionality to the sample size are shown along with the exact data.

\section{ACKNOWLEDGEMENT}

Financial support from the Australian Research Council (DP150103611) and the Government of Russian Federation (Grant 074-U01) is gratefully acknowledged.

\section{REFERENCES}

[1] J. B. Pendry, "Metamaterials: Going beyond nature," New Scientist, vol. 209, no. 2794, pp. ii-vii, 2011.

[2] P.-Y. Chen, M. Farhat, and A. Alu, "Bistable and self-tunable negative-index metamaterial at optical frequencies," Phys. Rev. Lett., vol. 106, no. 10, p. 105503, 2011.

[3] M. Lapine, I. V. Shadrivov, and Y. S. Kivshar, "Nonlinear metamaterials,” Rev. Mod. Phys., vol. 86, pp. 1093-1123, 2014.

[4] M. Lapine and S. Tretyakov, "Contemporary notes on metamaterials," IET Proc. Microwaves, Antennas and Propagation, vol. 1, no. 1, pp. 3-11, 2007.

[5] C. R. Simovski, "Analytical modelling of double-negative composites," Metamaterials, vol. 2, no. 4, pp. 169-185, 2008.

[6] J. Vehmas, S. Hrabar, S. Tretyakov, "Omega transmission lines with applications to effective medium models of metamaterials," J. Appl. Phys., vol. 115, p. 134905, 2014.

[7] C. R. Simovski, "On electromagnetic characterization and homogenization of nanostructured metamaterials," Journal of Optics, vol. 13, no. 1, p. 013001, 2011.

[8] M. Lapine, L. Jelinek, M. Freire, and R. Marqués, "Realistic metamaterial lenses: Limitations imposed by discrete structure," Phys. Rev. B, vol. 82, p. 165124, 2010.

[9] M. Lapine, L. Jelinek, and R. Marqués, "Surface mesoscopic effects in finite metamaterials," Opt. Express, vol. 20, no. 16, pp. 18297-18302, 2012.

[10] M. Lapine, L. Jelinek, R. Marqués, and M. Freire, "Exact modelling method for discrete finite metamaterial lens," IET Microw. Antenn. Propag., vol. 4, pp. 1132-1139, 2010.

[11] M. Gorkunov, M. Lapine, E. Shamonina, and K. H. Ringhofer, "Effective magnetic properties of a composite material with circular conductive elements," Eur. Phys. J. B, vol. 28, pp. 263-269, 2002.

[12] C. Menzel, , E. Hebestreit, R. Alaee, M. Albooyeh, S. Mühlig, S. Burger, C. Rockstuhl, C. Simovski, S. Tretyakov, F. Lederer, and T. Pertsch, "Extreme coupling: A route towards local magnetic metamaterials," Phys. Rev. B, vol. 89, p. $155125,2014$.

[13] M. Lapine, R. C. McPhedran, and C. G. Poulton, "Slow convergence to effective medium in finite discrete metamaterials," Phys. Rev. B, vol. 93, p. 235156, 2016. 